Size-selection initiation model extended to include shape and random factors

J. B. Trenholme, M. D. Feit, A. M. Rubenchik

November 8, 2005

Boulder Damage Symposium XXXVII

Boulder, CO, United States

September 19, 2005 through September 21, 2005 
This document was prepared as an account of work sponsored by an agency of the United States Government. Neither the United States Government nor the University of California nor any of their employees, makes any warranty, express or implied, or assumes any legal liability or responsibility for the accuracy, completeness, or usefulness of any information, apparatus, product, or process disclosed, or represents that its use would not infringe privately owned rights. Reference herein to any specific commercial product, process, or service by trade name, trademark, manufacturer, or otherwise, does not necessarily constitute or imply its endorsement, recommendation, or favoring by the United States Government or the University of California. The views and opinions of authors expressed herein do not necessarily state or reflect those of the United States Government or the University of California, and shall not be used for advertising or product endorsement purposes. 


\title{
Size-selection initiation model extended to include shape and random factors
}

\author{
John B. Trenholme*, Michael D. Feit, Alexander M. Rubenchik \\ University of California, Lawrence Livermore National Laboratory \\ P.O. Box 5508, L-463, Livermore, CA, USA 94550
}

\begin{abstract}
The Feit-Rubenchik size-selection damage model has been extended in a number of ways. More realistic thermal deposition profiles have been added. Non-spherical shapes (rods and plates) have been considered, with allowance for their orientation dependence. Random variations have been taken into account. An explicit form for the change of absorptivity with precursor size has been added. A simulation tool called GIDGET has been built to allow adjustment of the many possible parameters in order to fit experimental data of initiation density as a function of fluence and pulse duration. The result is a set of constraints on the possible properties of initiation precursors.
\end{abstract}

Keywords: Damage initiation, precursor, size selection model, laser damage

\section{INTRODUCTION}

For many years, laser damage researchers have been building models that improve understanding of the observed density of damage initiation sites as a function of laser beam fluence and pulse width. There is general agreement that initiation is caused by the violent destruction of unobservably small (nanometer size) absorbing precursors. These precursors are supposed to exist in large numbers, with only a small fraction contributing to the observed initiation density. The idea that damage properties could be produced from a model in which the size of the precursors was an important factor was put forward by Hopper and Uhlmann some time ago ${ }^{1}$. They assumed that linear absorption of laser energy raised the temperature of precursors until mechanical failure took place. They showed that there is a precursor size at which the temperature rise is maximized. Below that size, diffusion removes heat quickly. Above that size, the fact that absorption goes as the area, while thermal mass goes as the volume, leads to reduced temperature. A number of other workers extended their ideas ${ }^{2,3}$.

Hopper and Uhlmann followed most workers of the time in being most interested in the "threshold fluence" for laser damage. Below the threshold fluence, damage is supposed to be rare, while fluences above the threshold are sure to cause damage. More recently, experiments show that there is a steady increase in initiation density, per area or per volume, as the fluence is raised ${ }^{4}$. Feit and Rubenchik ${ }^{5}$ have extended the idea of size selection to allow for calculation of the curve of damage initiation density $\rho$ as a function of laser fluence $\phi$. Their basic idea was to find the range of precursor sizes which fail at any given fluence, and to assign to that fluence the number density of precursors between the smallest and largest failing sizes. They presumed that failure was due to runaway absorption above a critical temperature below the mechanical failure point. The latest results of their work are described in a companion paper ${ }^{6}$.

${ }^{*}$ To whom correspondence should be addressed: jbt@1lnl.gov; phone 925-422-6228 
This paper describes the extension of the size-selection model to allow for non-spherical inclusions, to generalize the absorption-versus-size assumptions and to add random factors. It describes a simulation tool that allows variation of the model parameters, with immediate visualization of the resulting $\rho(\phi)$ curve. The tool also finds the effect of pulse-width variation, and permits multi-parameter optimization to best fit experimental data.

\section{THE MODEL}

\subsection{Viewpoint}

The basic difference between the extensions discussed here and prior work is that we allow for a fraction $\mathbf{F}$ of the precursors at any given size to be initiated, with $\mathbf{F}$ somewhere between zero and unity. If $\mathbf{F}$ jumps abruptly from zero to unity at some small size, and jumps back to zero at some larger size, then the results of previous work are duplicated. More generally, however, we take the initiation density (per area or per volume) to be the integral, over size, of the number density of precursors (area or volume) $\mathbf{n}(\mathbf{s})$ times the initiation fraction $\mathbf{F}$ :

$$
\rho(\phi, \tau, \ldots)=\int_{0}^{\infty} n(s) F(s, \phi, \tau, \ldots) d s
$$

Since the quantities inside the integral are stochastic, it is a stochastic integral, meaning that results are not exact. Instead, the integral yields a mean value with some variation about the mean. We presume that values of $\mathbf{F}$ between zero and unity may be caused by orientation differences (for asymmetric particles), or by random variations in absorption, failure temperature, or other factors.

Note that the units of $\mathbf{n}(\mathbf{s})$, once size is integrated out, are carried through to become the units of $\rho(\phi)$. This means that the integral can be used to calculate surface damage density and volume damage density equally well.

\subsection{Assumptions}

We assume that:

- Absorbing precursors exist in large numbers, with a variety of sub-wavelength sizes

- The precursors absorb laser light, heat up, and the heat diffuses away

- Damage initiation happens when the hottest point in a precursor exceeds some critical temperature $\mathbf{T}_{\mathbf{x}}$

- Exceeding the critical temperature causes sharply increased absorption and thermal runaway, destroying a region larger than the precursor and leaving melted and fractured material behind

- The peak temperature that a precursor reaches depends on the size, shape, absorption profile and other characteristics of the precursor

- At low fluence, only some precursors will fail, at the hottest size (or shape, or absorption profile, or ...)

- As the fluence increases, a larger and larger fraction of the precursors will fail, over a broader range of sizes

\subsection{Simplifications}

In order to simplify the calculations, we make a number of additional assumptions. For the most part, these assumptions can be relaxed without causing serious changes in the results or conclusions: 
- Thermal properties are independent of temperature, until runaway (an average value is used)

- Precursor material and bulk material thermal properties are the same

- All materials are homogeneous and isotropic

- Absorption is constant inside the precursors, and zero outside

- Absorption is linear (single-photon)

- The precursors absorb only a small fraction of the incoming laser light (this is the "weak Beer's law" limit)

- Because of small absorption, deposition is near-uniform throughout the precursors

- Heat removal is only by thermal diffusion

- The only shapes we consider are simple ones for which we can do the diffusion math:

- Balls (3D diffusion)

- Rods (2D diffusion)

$\circ \quad$ Plates (1D diffusion)

\subsection{Precursor Number Density}

We take the number density $\mathbf{n}(\mathbf{s})$ of precursors (per area or per volume, per size) to have a power law form as a function of size $\mathbf{s}$ with exponent $\mathbf{Q}$, truncated at some minimum size $\mathbf{S}_{\mathbf{L O}}$ and also at some maximum size $\mathbf{S}_{\mathbf{H I}}$. If the total density of precursors (per area or per volume) is $\mathbf{N}$, then the density is given by

$$
n(s)=N \frac{(Q-1)}{\left(s_{L O}^{1-Q}-s_{H I}^{1-Q}\right) s^{Q}} \quad s_{L O}<s<s_{H I}
$$

We use this functional form because it is the type of variation often observed in natural processes such as optics contamination, laboratory dust, and so forth. Common values of $\mathbf{Q}$ are in the range from 1 to 3 . We insert the lower size limit to avoid getting infinite density when we integrate down to zero size. This is reasonable because there is a physical lower size limit, since precursors of nanometer size are only a few molecules across. The upper size limit is just a convenience in numerical computations, since (as we shall see) it has very small effect on the results of the model. Also, large particles (say a few microns or more) would be visible in optical inspection unless their absorption was extremely low.

\subsection{Laser Beam Absorption}

In principle, the absorption of laser light by the precursors could be quite complex. Although they are sub-wavelength in size, diffraction might be important. We would then see resonances as a function of size and shape, and calculation would be difficult. To investigate this issue, we calculated absorption by small balls with a small absorptive part of their refractive index, using the exact Mie theory ${ }^{7}$. We found that the absorption profile inside the balls was almost exactly what one would calculate by using absorption along undeviated rays (this is not true for metallic inclusions - see Figure 4 in Walker ${ }^{2}$ et. al.). Although there were strong resonances outside the balls when the real parts of the refractive index were different in the ball and the substrate, perturbations from the simple ray picture were small inside, and largely confined to the output surface. We therefore used the straight-ray results for balls, and assumed that the same undeviatedray picture could be used with plates and rods as well.

When absorption in the precursors is weak (as we shall see it must be) and is found from an undeviated-ray picture, the deposition inside a uniform-absorption-coefficient precursor is close to spatially uniform. We therefore adopted this picture. To test its validity, we carried out finite-element calculations of temperature rise in balls for nonuniform absorption coefficients and large absorption coefficients. With appropriate 
scaling, the peak temperatures reached were very close to those found from weak absorption of undeviated rays, even with 3 e-foldings of irradiance across the ball.

When there is no diffusion, the rate of temperature rise due to weak absorption of undeviated rays in any shape is given by

$$
\frac{d T}{d t}=\frac{I \beta}{C_{V}}
$$

Here $\mathbf{T}$ is the temperature rise above the initial temperature, $\mathbf{I}$ is the laser beam irradiance, $\boldsymbol{\beta}$ is the absorption coefficient inside the precursor, and $\mathbf{C}_{\mathbf{V}}$ is the specific heat per unit volume (recall that $\boldsymbol{\beta}$ and $\mathbf{C}_{\mathbf{V}}$ are supposed to be average values over the range of expected temperatures from ambient to $\mathbf{T}_{\mathbf{x}}$ ). Taking some representative values, assume that $\mathbf{I}=1 \mathrm{GW} / \mathrm{cm}^{2}, \boldsymbol{\beta}=10^{5} \mathrm{~m}^{-1}\left(0.1\right.$ per micron) and $\mathbf{C}_{\mathbf{V}}=2 \mathrm{~J} / \mathrm{cc} /{ }^{\circ} \mathrm{K}$ (typical of $\mathrm{SiO}_{2}$ or KDP). The temperature would then rise at $500{ }^{\circ} \mathrm{K} / \mathrm{ns}$. We see that the absorption coefficient cannot be much smaller than the value we assumed, as temperatures high enough to initiate damage would not be reached during the duration of typical laser pulses. Likewise, the absorption coefficient cannot be much larger than the assumed value, since temperatures high enough to form plasma would be reached in less than a nanosecond. This is the reasoning behind the remark that absorption coefficients must be so small that absorption in a sub-wavelength precursor is small.

\subsection{Thermal Diffusion}

We are assuming that thermal diffusion reduces the temperature found in the above nodiffusion result. The diffusion equation (using the thermal source above) is

$$
\frac{\partial T}{\partial t}=\frac{I \beta}{C_{V}}+\nabla \bullet(D \nabla T)
$$

Here $\mathbf{D}$ is the diffusion coefficient, again using a temperature-averaged value to maintain linear behavior.

It is a straightforward exercise ${ }^{8}$ to find the temperature in plate-, rod- and ball-shaped regions, embedded in an infinite substrate, that are raised by a delta-function thermal input that causes unit temperature rise at time zero, with no subsequent thermal input. Suppose the regions to have a size $\mathbf{s}$ (this is the half-thickness in the case of the plate, and the radius for rods and balls). The peak temperature is always at the center point of the regions, and is given by

$$
T_{1}=\operatorname{erf}\left(\frac{1}{\sqrt{v}}\right) \quad T_{2}=1-e^{-1 / v} \quad T_{3}=\operatorname{erf}\left(\frac{1}{\sqrt{v}}\right)-\frac{2 e^{-1 / v}}{\sqrt{\pi v}}
$$

We have introduced a normalized time $\mathbf{v}$, given by the actual time divided by the diffusion time for a distance $\mathbf{s}$ :

$$
v=\frac{t}{\tau}=\frac{4 D t}{s^{2}}
$$

The subscript on the temperatures indicates the dimensionality of the regions: 1 is for plates, 2 is for rods and 3 is for balls.

\subsection{Laser Pulse Shape}

We now have the impulse responses of the various precursor geometries, and since we have imposed linearity we can convolve the impulse responses with the temporal shape of the laser irradiation to get the peak temperature in the precursors as a function of time. The results are available in terms of standard functions in the case of flat-in-time laser irradiation that jumps from zero irradiance at time zero to a fixed level, and then 
maintains that level indefinitely. Linearity also means that we can superimpose multiple step functions of this type to simulate more complicated pulse shapes. It turns out that the response to a ramp starting at time zero can also be found in terms of the same standard functions, so we could do arbitrary piecewise-linear pulse shapes. In this paper, we will restrict ourselves to flat-in-time (FIT) laser irradiance temporal shapes. For FIT pulses, the peak temperature in time is always at the end of the pulse, so we do not have to search for it. The temperature as a function of normalized time is given by

$$
T_{1}=\frac{\beta \phi}{C_{V}}\left[1-\left(1+\frac{2}{v}\right) \operatorname{erfc}\left(\frac{1}{\sqrt{v}}\right)+\frac{2}{\sqrt{\pi v}} e^{-1 / v}\right] \quad T_{2}=\frac{\beta \phi}{C_{V}}\left[1-e^{-1 / v}+\frac{E_{1}\left(\frac{1}{v}\right)}{v}\right] \quad T_{3}=\frac{\beta \phi}{C_{V}}\left[1-\left(1-\frac{2}{v}\right) \operatorname{erfc}\left(\frac{1}{\sqrt{v}}\right)-\frac{2}{\sqrt{\pi v}} e^{-1 / v}\right]
$$

Here $\phi$ is the fluence in the laser pulse, and $E_{1}$ represents the exponential integral. The term in front of the square brackets is the temperature rise in the absence of diffusion, and the quantities inside the square brackets are the diffusion factors that reduce the temperature. We will call these diffusion factors $\mathbf{g}_{\mathbf{J}}(\mathbf{v})$, where $\mathbf{J}$ is the dimensionality of the diffusion (1, 2 or 3 for plates, rods or balls). For times small compared to the diffusion time all the diffusion factors are unity. For times much larger than the diffusion time, the peak temperature in the plate increases as $V_{t}$ and that in the rod increases as $\ln (\mathrm{t})$. The peak ball temperature, however, asymptotically approaches a constant value equal to the no-diffusion temperature that would be caused by constant laser irradiation over a time equal to twice the diffusion time.

In the forms shown above, the diffusion factors are somewhat difficult to use due to cancellation of terms, and so we have found numerical approximations that are both speedy and accurate (to $0.1 \%$ relative error) for short and long times.

\subsection{Precursor Failure Fluence for Different Shapes}

Precursors have been assumed to fail at some temperature rise $\mathbf{T}_{\mathbf{x}}$. Equating this to the spatial maximum temperature rise at the end of a FIT pulse, and introducing the critical fluence $\phi_{\mathrm{C}}$ at which the precursor fails, we have

$$
T_{x}=\frac{\beta \phi_{C}}{C_{V}} g_{J}(v) \Rightarrow \phi_{C}=\frac{T_{x} C_{V}}{\beta g_{J}(v)}
$$

We immediately see that this model (or any similar model) will depend only on the grouping $\mathbf{T}_{\mathbf{x}} \mathbf{C}_{\mathbf{V}} / \boldsymbol{\beta}$, and not on any of its factors individually. We may reasonably expect that $\mathbf{C}_{\mathbf{V}}$ will be close to the specific heat of the bulk material, even if (contrary to our assumption) $\mathbf{C}_{\mathbf{V}}$ is somewhat different in the precursor, since much of the thermal diffusion takes place in the bulk rather than the precursor. But there will be no way to extract the critical temperature $\mathbf{T}_{\mathbf{x}}$ or absorption coefficient $\boldsymbol{\beta}$ individually by comparing this model to experiment. Only their ratio can be determined.

For times short compared to the diffusion time, all the diffusion factors are unity and the failure fluence is given by

$$
\phi_{C}=\frac{T_{x} C_{V}}{\beta} \quad t<<\frac{s^{2}}{4 D}
$$

The failure fluence, in this short-pulse limit, is independent of pulse width. For times long compared to the diffusion time, we can use the results in section 2.7 to see that the ball failure fluence increases linearly with the time $t$, the rod failure fluence increases as $\mathrm{t} / \ln (\mathrm{t})$, and the plate failure fluence increases as $\sqrt{ } \mathrm{t}$. 


\subsection{Relation between Size and Absorption Coefficient}

We do not expect the critical temperature or specific heat to depend strongly on precursor size. However, the absorption coefficient may well depend on size, and so we incorporate such a dependence into the model.

Consider, for example, the absorption-versus-size relations we would get under various scenarios. Suppose that ball-shaped precursors form from extremely small bits of contaminant. These small bits spread out by diffusion of their material during substrate creation to make balls of different sizes, all with the same amount of contaminant. If the absorption coefficient is linearly proportional to contaminant density, then the absorption coefficient in the balls is proportional to the inverse cube of their size. Similar reasoning, using initial thread-shaped sources, leads to inverse square dependence for rods. Plates arising from initial thin foil sources would have absorption coefficients proportional to the inverse of their size.

In the other extreme, if all the precursors were formed of identical material with no change of concentration with size, the absorption coefficient would be independent of size (size to the power zero).

To allow for these and many other possibilities, we assume a power-law dependence of absorption coefficient on size, using an exponent $\mathbf{B}$. This dependence does not have to hold over a broad range of sizes, since (as we shall see in section 2.12) only a fairly small range of sizes contributes to initiation. It is sufficient if the behavior looks like a power law over one, or perhaps two, decades of size variation. We introduce an arbitrary reference size $\mathbf{s}_{\mathbf{0}}$ and the absorption coefficient $\boldsymbol{\beta}_{\mathbf{0}}$ at that size, and write the absorption coefficient as

$$
\beta=\beta_{0}\left(\frac{s}{s_{0}}\right)^{B}
$$

Only one of the two parameters $\mathbf{s}_{\mathbf{0}}$ and $\boldsymbol{\beta}_{\mathbf{0}}$ is needed here; we have introduced two for convenience. Normally we will take $\mathbf{s}_{\mathbf{0}}$ to be fixed at some arbitrary size, and vary only $\mathbf{B}$ and $\boldsymbol{\beta}_{0}$ to fit data values.

\subsection{Failure-Fluence Size Scaling for Different Shapes}

Inserting the power-law absorption coefficient scaling into the equation for failure fluence, we have an expression for the failure fluence as a function of geometry and physical parameters

$$
\phi_{J}=\frac{T_{x} C_{V}}{\beta g_{J}(v)}=\frac{T_{x} C_{V}}{\beta_{0}\left(\frac{s}{s_{0}}\right)^{B} g_{J}\left(\frac{4 D t}{s^{2}}\right)}
$$

Once again, $\mathbf{J}$ is the dimensionality of the diffusion (1,2 or 3 for plates, rods or balls).

In order for size selection to take place, we want the failure fluence to increase as the size decreases. It is not as important for the failure fluence to decrease for large sizes, since the rapid drop-off of number density with size will cut the integral off at the largeprecursor end unless the failure fluence drops off very sharply there (faster than the number density is decreasing). The desire for failure fluence increase at small sizes sets a lower limit on the absorption coefficient scaling power $\mathbf{B}$. For plates, the lower limit is $\mathbf{B}>-1$. For rods, the lower limit is $\mathbf{B}>-2$. For balls, the lower limit is also $\mathbf{B}>-2$. For all three shapes, failure fluence will increase at large sizes if $\mathbf{B}<0$. Some representative 
curves of relative failure fluence as a function of relative size are shown in Figures 1, 2 and 3 (the numbers on the curves are $\mathbf{B}$ values). Note that there is a minimum in the curves, corresponding to size selection at both small and large sizes, only for values of $\mathbf{B}$ between the lower limits given above and zero.
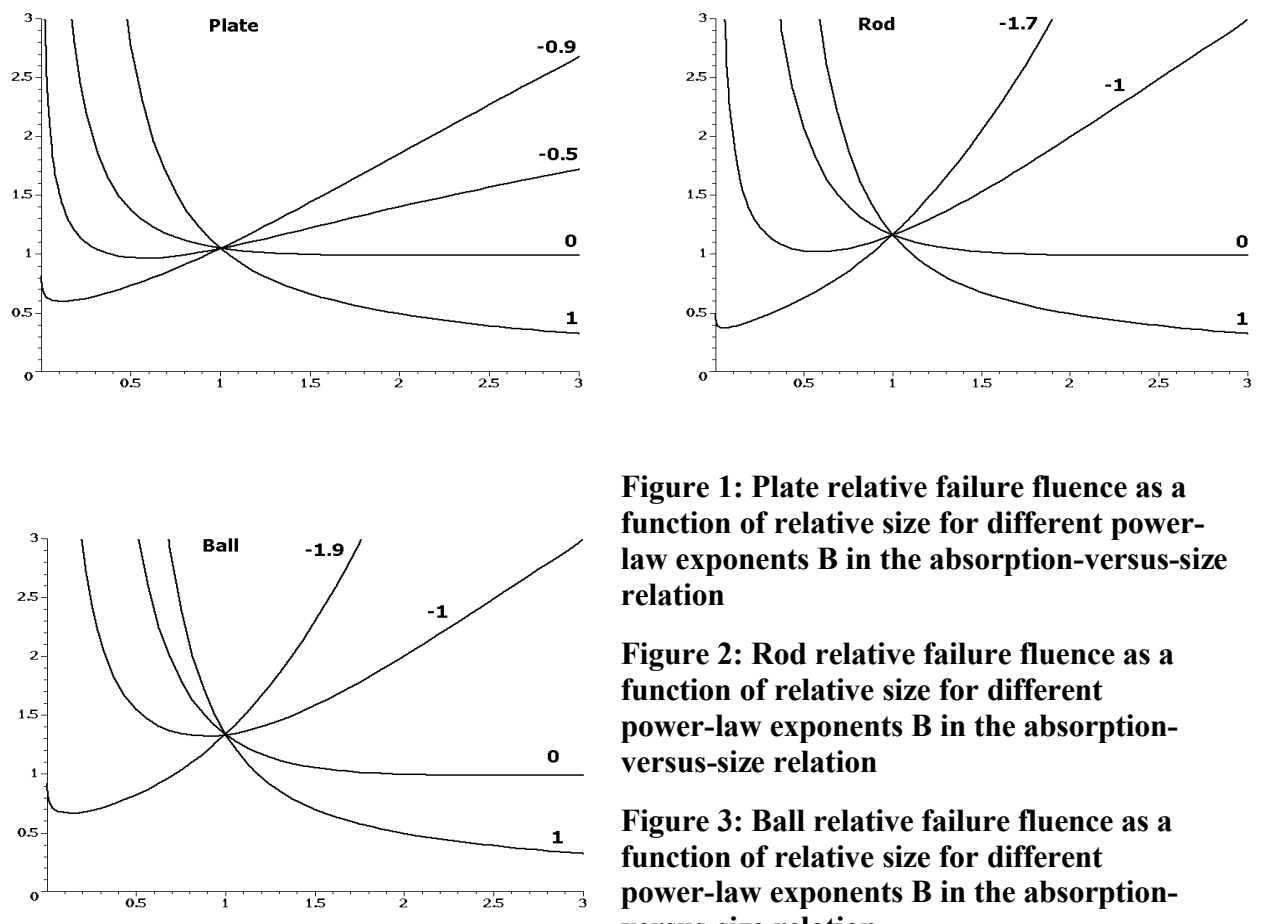

Figure 1: Plate relative failure fluence as a function of relative size for different powerlaw exponents $B$ in the absorption-versus-size relation

Figure 2: Rod relative failure fluence as a function of relative size for different power-law exponents $B$ in the absorptionversus-size relation

Figure 3: Ball relative failure fluence as a function of relative size for different power-law exponents $B$ in the absorptionversus-size relation

\subsection{Plate and Rod Orientation}

Up to this point, we have ignored the orientation of the precursors, and calculated heating for the hottest-case orientation. For balls, there is no orientation factor. However, rods and plates heat up at rates that depend on their angle with respect to the laser beam. If we define a unit vector normal to the plate surface, and a unit vector aligned along the rod axis, then the deposition rate in a plate is proportional to the absolute value of the cosine of the angle between the orientation vector and the laser beam direction, while the deposition rate in a rod is proportional to the absolute value of the sine of the angle. We presume here that the orientation vectors are random on the sphere of possible directions (non-uniformity can lead to an orientation-dependent initiation rate for the optic). The fraction of precursors reaching the critical temperature is a function of the ratio $\mathbf{h}$ of initiation fluence to minimum initiation fluence at the most-strongly-heated orientation. This ratio depends on the precursor shape; it is given by: 


$$
h_{J}=\frac{\phi \beta_{0}\left(\frac{s}{s_{0}}\right)^{B} g_{J}\left(\frac{4 D t}{s^{2}}\right)}{T_{x} C_{V}}
$$

The failing fraction is zero for all three geometries for $\mathbf{h}$ less than unity. Above $\mathbf{h}=1$, the plate, rod and ball fractions are given by

$$
F_{1}=1-\frac{1}{h} \quad F_{2}=\sqrt{1-\frac{1}{h^{2}}} \quad F_{3}=1 \quad h>1 \quad h=\frac{\phi_{I N I T}}{\phi_{C}}
$$

The shapes of these functions are shown below.

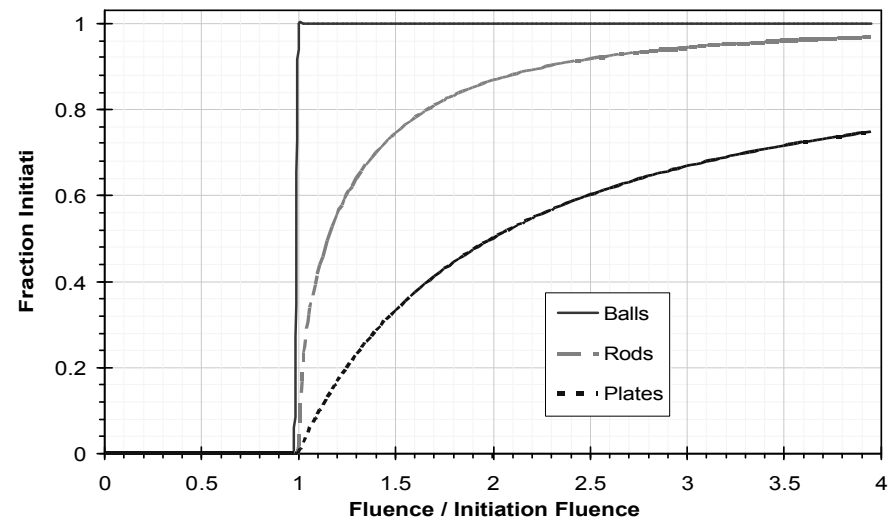

Figure 4: Curves of cumulative fraction initiating due to random orientation for the three shapes

The addition of an orientation dependence means that the curves shown in Figures 1 and 2 are to be taken as the lower limit of initiation, with larger and larger fractions of randomly oriented precursors initiating at fluences above the curves. The ball curves in Figure 3 are transition values - above the curves, all precursors have initiated.

\subsection{Random Variations}

Precursors are not likely to be identical. Aside from variations of size and geometry, we expect that there will be individual variations of absorption coefficient, critical temperature rise and other factors. We introduce such variations into the model by smearing out the initiated-fraction curves over a range of fluences. We want the curves to remain in positive-fluence territory, and so we want to convolve the curves with a random distribution that exists only for positive values of its argument. We somewhat arbitrarily choose to use the log-normal distribution. This distribution is close to Gaussian when the standard deviation is small compared to the mean, but skews to the positive side for large standard deviations and never goes negative.

For mathematical convenience, it is better to do the convolution calculations in terms of the variable $\mathbf{c}$ that is the reciprocal of $\mathbf{h}$. The log-normal is now avoiding "wrapping around infinity" at the low end, and still cannot get to negative fluence at the high end, and the plate convolution becomes tractable.

For balls, the convolution is of the CDF of a log-normal with two delta functions. The resulting initiated fraction distribution is just a cumulative log-normal distribution. Written in terms of $\mathbf{h}$, it is given by 


$$
F_{3}=\frac{1}{2} \operatorname{erfc}\left(\frac{\ln \left(\frac{h}{\sqrt{1+z^{2}}}\right)}{\sqrt{2 \ln \left(1+z^{2}\right)}}\right)
$$

Here $\mathbf{z}$ is the ratio of standard deviation to mean (sometimes called the "contrast"). It measures the amount of random variation, with $\mathbf{z}=0$ corresponding to no randomness (orientation factors only).

The convolution integral for plates is more difficult, but it can still be evaluated, using the fact that the orientation PDF is unity from $\mathbf{c}=0$ to $\mathbf{c}=1$. The result is equal to the ball distribution above plus an adjustment term:

$$
F_{1}=\frac{1}{2} \operatorname{erfc}\left(\frac{\ln \left(\frac{h}{\sqrt{1+z^{2}}}\right)}{\sqrt{2 \ln \left(1+z^{2}\right)}}\right)-\frac{1+z^{2}}{2 h} \operatorname{erfc}\left(\frac{\ln \left(\frac{h}{\left(1+z^{2}\right)^{3 / 2}}\right)}{\sqrt{2 \ln \left(1+z^{2}\right)}}\right)
$$

The convolution integral for rods appears to be intractable. We therefore approximate the rod result by using the square root of the plate result with $\mathrm{h}$ replaced by its square, and $\mathrm{z}$ increased by $\sqrt{2}$. This makes the result exact for no random component (see the orientation distributions above), and the $\mathrm{z}$ adjustment makes the added random part correct near $\mathrm{h}=1$ for small values of $\mathrm{z}$. For large $\mathrm{z}$, the result deviates somewhat from the results of a numerical convolution, but this happens for $\mathrm{z}$ values so large (comparable to unity, or even greater) that the initial assumption of log-normal noise might well be questioned. Given these caveats, we use the equation

$$
F_{2}=\sqrt{\frac{1}{2} \operatorname{erfc}\left(\frac{\ln \left(\frac{h^{2}}{\sqrt{1+2 z^{2}}}\right)}{\sqrt{2 \ln \left(1+2 z^{2}\right)}}\right)-\frac{1+2 z^{2}}{2 h^{2}} \operatorname{erfc}\left(\frac{\ln \left(\frac{h^{2}}{\left(1+2 z^{2}\right)^{3 / 2}}\right)}{\sqrt{2 \ln \left(1+2 z^{2}\right)}}\right)}
$$

The effect of random variation can be seen in Figure 5, where the ball initiated fraction $\mathrm{F}_{3}$ (vertical axis) is seen on the left with only a small random addition, and on the right with a large random component. With little randomness, the initiated fraction is mostly either zero or unity, but random variation gives us a large region with intermediate values. 

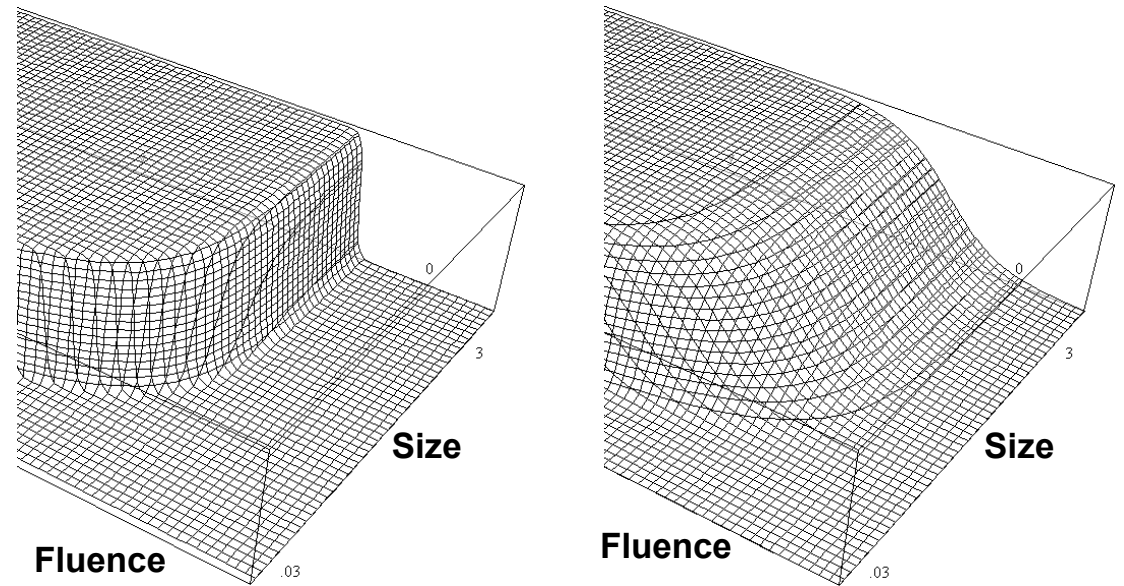

Figure 5: Effect of random variation on initiation fraction for ball shapes - $B=0$

\subsection{Integration over Precursor Size}

We now have everything we need to evaluate the initiation density as a function of fluence. In Figure 6, the product of the number density (for $\mathrm{Q}=2$ ) times the initiated fraction (for ball shapes with moderate randomness) is seen as a function of size and fluence. A heavy line illustrates the path of integration for one fluence value. We see that the drop-off of precursor number density as size increases concentrates the integrand along the small-size edge of the initiation region. Although precursors of all sizes within the initiation region are failing, most of the initiation density comes from the smaller precursors in the region, since they are more numerous. As we raise the fluence, we get an increased initiation density largely because the initiation region moves to lower sizes, where there are more available precursors.

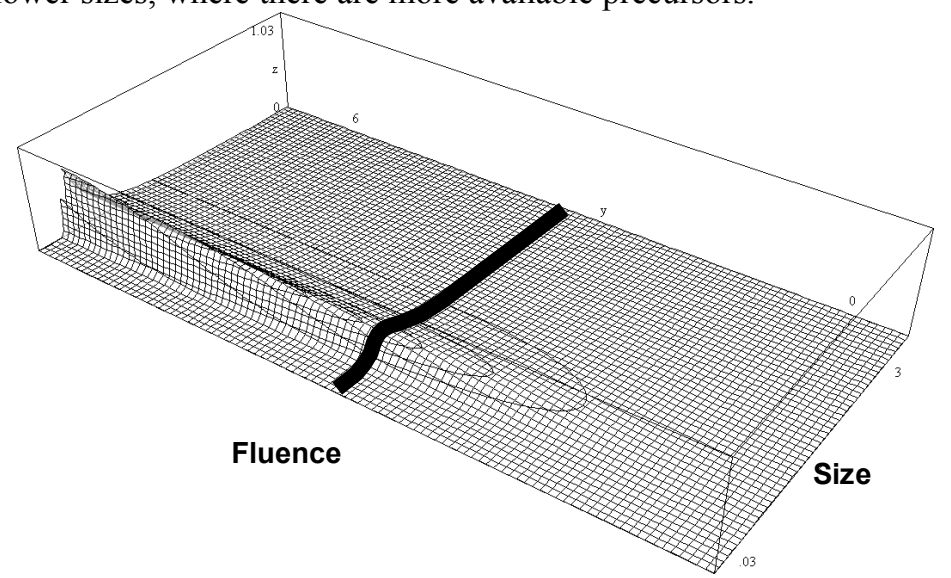

Figure 6: Integrand of the initiation density integral. Heavy line shows the integration path for one fluence value

\section{GIDGET}

\subsection{Overview}

GIDGET (Get Initiation Density via Globule Explosion Temperature) is a spreadsheet that implements the calculations that make up the model described above. It is designed to allow variation of the parameters of the model, produce the resulting curve of initiation density as a function of fluence, and allow comparison of the curve with experimental data. Using the multi-parameter optimization feature available with the spreadsheet, parameters can be automatically adjusted for best fit. 


\subsection{Routines for Integration over Precursor Size}

Most of the functions needed for GIDGET are supplied by the spreadsheet program. However, there are some routines that must be supplied by the user. These routines have been implemented in the "macro" language of the spreadsheet. The routines include:

- An adaptive integration routine. It is used because fixed-interval integration is very inefficient when the integrand is peaked in one small region of the entire range of integration. The routine uses Simpson integration, subdividing high-contribution regions recursively to meet a specified error tolerance.

- The integrand function called by the adaptive integrator. This routine contains:

- Tests to select an execution path depending on the geometric shape of the precursors.

- Fast approximations to the $\mathrm{gJ}(\mathrm{v})$ diffusion functions. These are polynomial ratios (Padé forms).

- Calculations of the combined effects of orientation and random factors.

\subsection{Matching the Pulse Width Variation of Initiation Fluence}

We want to match the variation of damage initiation fluence with pulse width. Typically, initiation fluence (for constant initiation density) varies as a power of the pulse duration $\tau$. Typical values are in the range $\tau^{0.35}$ to $\tau^{0.5}$. We chose to start with a reference pulse duration, and then to calculate the initiation density for another pulse duration. The fluence at the new duration is set to give the same initiation density as at the reference duration when the model gives the same scaling as experiment. Calling the reference pulse width $\tau_{1}$ and the new pulse duration $\tau_{2}$, and taking the pulse-width-scaling exponent to be $\mathbf{P}$, we used the model to calculate

$$
\rho\left(\phi_{1}\left(\frac{\tau_{2}}{\tau_{1}}\right)^{P}, \tau_{2}, \ldots\right) \cong \rho\left(\phi_{1}, \tau_{1}, \ldots\right)
$$

Equality is achieved when the model correctly scales with exponent $\mathbf{P}$. The model initiation density is found at each data-point fluence, and compared to values found from the above formula, using $\tau_{2}$ values of $1 / 3$ of $\tau_{1}$ and 3 times $\tau_{1}$.

\subsection{Comparing the Model to Experimental Data}

The model as described here calculates single-shot (unconditioned) initiation levels (extension to ramped fluence and conditioning is discussed in the companion paper ${ }^{6}$ ). We have therefore used single-shot data taken on fused silica exit surface damage as a test case. The data were taken with Gaussian pulses, but the model presently uses flat-intime pulses; we presumed that the correction was the same at all fluences. The pulsewidth scaling power was taken as 0.37 , in agreement with experiment. The diffusion coefficient was fixed at $0.01 \mathrm{~cm}^{2} / \mathrm{s}$, which is typical of $\mathrm{SiO}_{2}$ (and KDP). The precursor shape was set to "ball." The spreadsheet nonlinear optimizer was then asked to adjust the absorption parameters $\mathbf{B}$ and $\boldsymbol{\beta}_{\mathbf{0}}$, the precursor density parameters $\mathbf{N}, \mathbf{Q}$ and $\mathbf{s}_{\mathbf{L O}}$, and the random component $\mathbf{z}$ for best fit to the data. The fit error was calculated as the sum of the square of the relative errors, since the data varies over several orders of magnitude and we want to fit both the low end and the high end equally well. The minimum-error result is shown in Figure 7. We see that the data (large circles) is reasonably well fit by the model (solid line), once the parameters are adjusted. For comparison, a reference curve with power law dependence (exponent 3.9 in this case) is also shown on the plot (dashed line). It agrees well with the model, except at the low-fluence end. We have no simple explanation for this agreement. Also shown are the shorter-pulse-duration points $(+)$ and longer-pulse-duration points $(\mathrm{x})$, which are seen to come at the same initiation density values as the model curve, demonstrating correct pulse-width dependence. 

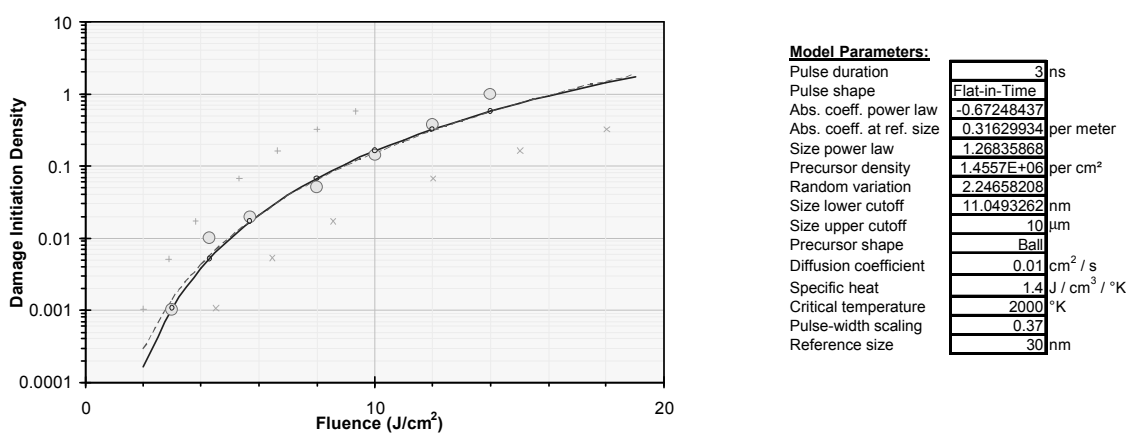

Figure 7: Result of GIDGET fitting output-surface $\mathrm{SiO}_{2}$ damage data by variation of six parameters. See text in section 3.4 for a description of the details in the plot.

\subsection{Extensions to the Model}

It is straightforward to add conditioning to the model, using the method described in the companion paper $^{6}$. It is also easy to add multiple-population precursors, since the initiation density for two independent populations is just the sum of their individual initiation rates. Precursor shapes other than those used here can be added once their diffusion reduction functions and orientation dependence are specified. The extension to arbitrary piecewise-linear laser pulse shapes was mentioned previously; it requires the addition of one diffusion expression for each temporal segment, and a way to find the maximum temperature as a function of time (any standard univariate extremum-finding method should work). The model as implemented is of satisfactory speed (optimization over 6 parameters, with a hundred or more trial parameter sets, takes a minute or less on a $2 \mathrm{GHz}$ PC) but the addition of some of these features will probably require the linking of compiled $\mathrm{C}++$ code to the spreadsheet to maintain satisfactory speed.

\section{CONCLUSIONS}

The addition of non-spherical precursor shapes, variation of absorption coefficient with size, and random factors has increased the flexibility of the size-selection model of damage initiation as a function of fluence. Implementation of the improved model in a spreadsheet has allowed rapid examination of the effect of parameter changes. Use of the multi-parameter optimization feature of the spreadsheet gives us the ability to adjust multiple parameters for the best fit to data sets. The increase in both flexibility and speed allows us to examine the implications of the model quickly and easily, and to compare the model to numerous experiments.

\section{ACKNOWLEDGEMENTS}

The authors have benefited greatly from the encouragement and direction of Mary Spaeth.

This work was carried out in support of the laser modeling activity of the National Ignition Facility Project.

This work was performed under the auspices of the U. S. Department of Energy by the University of California, Lawrence Livermore National Laboratory under Contract No. W-7405-ENG-48.

\section{REFERENCES}


${ }^{1}$ R. W. Hopper and D. R. Uhlmann, "Mechanism of Inclusion Damage in Laser Glass", J. Appl. Phys. 41, 4023-4037 (1970)

${ }^{2}$ T. W. Walker, A. H. Guenther and P. Nielsen, "Pulsed Laser-Induced Damage to ThinFilm Optical Coatings - Part II: Theory”, IEE J. Quantum Electron., QE-17, 2053-2065 (1981)

${ }^{3}$ M. R. Lange, J. K. McIver, A. H. Guenther and T. W. Walker, "Pulsed Laser Induced Damage of an Optical Material with a Spherical Inclusion: Influence of the Thermal Properties of the Materials", Laser Induced Damage in Laser Materials 1982, NBS Spec. Pub. 669, 380-386 (1984)

${ }^{4}$ M. D. Feit et. Al., "Extrapolation of damage test data to predict performance of largearea NIF optics at $355 \mathrm{~nm}$ ", Laser-Induced Damage in Optical Materials: 1998, Proc. SPIE 3578, 226-231 (1999)

${ }^{5}$ M. D. Feit and A. M. Rubenchik, "Implications of nanoabsorber initiators for damage probability curves, pulselength scaling and laser conditioning", Symposium on Optical Materials for High Power Lasers 2003, Proc. SPIE 5273, 74 (2003)

${ }^{6}$ M. D. Feit, A. M. Rubenchik and J. B. Trenholme, "Simple model of laser damage initiation and conditioning in frequency conversion crystals", these proceedings

${ }^{7}$ We used a slightly modified, double-precision version of the S7 code described in P. W. Barber and S. C. Hill, Light Scattering by Particles: Computational Methods, World Scientific, 1990. S7 is available at http://atol.ucsd.edu/ pflatau/

${ }^{8}$ See, for example, H. S. Carslaw and J. C. Jaeger, Conduction of Heat in Solids (second edition), Oxford University Press, sections 2.2 and 10.3 